\title{
RECENT ADVANCES IN ATOMIC MODELING
}

\author{
W. H. Goldstein ${ }^{1}$ \\ ${ }^{1}$ High Temperature Physics Division, Lawrence Livermore National Laboratory, \\ Livermore, CA 94550 USA
}

\begin{abstract}
Precision spectroscopy of solar plasmas has historically been the goad for advances in calculating the atomic physics and dynamics of highly ionized atoms. Recent efforts to understand the laboratory plasmas associated with magnetic and inertial confinement fusion, and with X-ray laser research, have played a similar role. Developments spurred by laboratory plasma research are applicable to the modeling of high-resolution spectra from both solar and cosmic X-ray sources, such as the photoionized plasmas associated with accretion disks. Three of these developments in large scale atomic modeling are reviewed: a new method for calculating large arrays of collisional excitation rates, a sum rule based method for extending collisional-radiative models and modeling the effects of autoionizing resonances, and a detailed level accounting calculation of resonant excitation rates in FeXVII.
\end{abstract}

\section{INTRODUCTION}

The possibility of creating hot, dense, earthbound plasmas using powerful lasers and pulsed power sources has opened new avenues of research into the atomic physics of highly ionized atoms and their behavior under a broad range of plasma conditions. High resolution spectroscopy of these sources has posed significant new challenges to modelers of the atomic kinetics of non-equilibrium (non-LTE) plasmas. In particular, neither coronal nor Saha equilibrium typically obtains, and the application of detailed collisionalradiative physics is essential.

The collisional-radiative regime yields little simplification in atomic kinetics. States and processes that could be neglected in coronal equilibrium cannot be ignored. Excited atomic levels may be populated by recombination, ionization and photo-driven processes, in addition to simple collisional excitation. Ionization equilibrium cannot be assumed. Line formation mechanisms in astrophysical plasmas also run a wide gamut. The standard collisional models, appropriate for solar coronal plasmas, are not applicable to recombining, photo-ionized sources like accretion disks. As high resolution spectral data from cosmic plasmas becomes available, the need will arise for more suitable modeling tools.

In this paper I'll review three advances in collisional-radiative modeling: an improvement in computational efficiency; an improvement in the approximate treatment of kinetics; and, the converse, improvements in the level of detail. My discussion of the first two topics will be limited to theoretical notes and brief comments on phenomenological applicability. Under the third heading I'll present results of a new calculation, in a detailed level accounting scheme, of resonant excitation rates in FeXVII. 


\section{THE PAINLESS WAY TO ACCURATE, RELATIVISTIC, MULTICONFIG- URATIONAL, DISTORTED WAVE COLLISIONAL EXCITATION RATES}

Electron densities well above $10^{19} \mathrm{~cm}^{-3}$ are easily achieved in vacuum spark and laserproduced plasmas. Though still far from LTE (the high density limit where atomic dynamics become irrelevant), these conditions cannot be treated in the coronal approximation. Rather, it is necessary to account for multiple collisional excitations, and collisional, as well as radiative, deexcitations.

For a perspective on this complication, consider a model for the neon-like ion. From its $1 s^{2} 2 s^{2} 2 p^{6}$ ground state, we can construct the 36 lowest lying excited states of this ion by promoting an electron out of the $n=2$ shell and into the $n=3$ shell. In the coronal limit, a kinetics model of this ion would require the calculation of 36 collisional excitation rates (in addition to the cascade matrix). Because of the low density, an excited state, once created by a collision, decays radiatively long before the ion can undergo a second collision. However, at high density, excited states are collisionally coupled to each other, as well as to the ground state, so that $(37 \times 36) / 2=666$ excitation rates are required.

The problem is that accurate collisional excitation rates are computationally expensive - until recently it took anywhere from one half to several hours (K. Reed, private communication; Goldstein and Reed 1986) of Cray CPU time to obtain just the 36 rates needed for the coronal model in the relativistic distorted wave scheme. (To my knowledge, no benchmark existed for the 666 rate high density case.) An elegant solution to this problem was recently discovered by computational atomic physicists at Hebrew University (Bar-Shalom, Klapisch and Oreg 1988). Their solution is based on a powerful factorization theorem, combined with an empirically motivated interpolation approximation.

The factorization theorem is an exact formula for separating a collisional excitation cross section into an angular recoupling coefficient and a radial part.(Oreg 1971, 1975; Bar-Shalom et al. 1988) The former subsumes the details of the target state angular couplings, and is entirely independent of the dynamics of the interaction and the kinematical variables used for its description. The radial factor is a sum over partial waves, and depends on the bound orbitals involved in the transition as well as the continuum electron's energy and wave function, but is independent of the specific atomic states. Thus, it is formally common to many level-to-level transitions, and need not be recalculated for each one. (This result may sound suspiciously like the Wigner-Eckart theorem, but note that we're talking about factorizing cross sections -- including interference and exchange terms and a partial wave sum -- not just matrix elements.)

Factorization is not the whole story, though, since the radial components for each transition are not completely independent of the detailed levels involved. The dependence is indirect, arising from the change in energy of the continuum electron, i.e., the threshold energy, $\Delta \mathrm{E}$. But this dependence is smooth, and for sufficiently small changes in threshold energy, the dependence can be treated, to arbitrary accuracy, by linear interpolation. In fact, by performing many calculations, the Hebrew University group determined empirically that, when the energy of the outgoing electron is held fixed, the dependence of the radial factor on $\Delta \mathrm{E}$ (or, in the case of dipole-allowed transitions, $\log \Delta \mathrm{E}$ ) is linear over a quite large range (Bar-Shalom et al. 1988). 
Since the radial integrals represent the largest expense in calculating cross sections, the factorization-interpolation solution yields tremendous savings in computational effort. For example, far from taking on the order of an hour for the 36 rate neon-like problem, this method requires about 4 minutes in Cray CPU time to obtain the 666 rates needed under non-coronal conditions, with no significant loss in accuracy (Goldstein and Reed 1986). Figure 1 should help explain whence this savings comes, as well as demonstrate an application to the fluorine-like ion. This charge state has 111 low-lying excited levels of the form $1 s^{2} 2 s^{1} 2 p^{6}$ and $1 s^{2}(2 s 2 p)^{6} 3 l, l=s, p, d$, and even the coronal problem of generating all 111 ground state excitation rates is daunting. As shown in Figure 1, eight of these rates describe excitation from the $1 s^{2} 2 s^{2} 2 p^{5}(J=3 / 2)$ ground level to the levels belonging to the $1 \mathrm{~s}^{2} 2 \mathrm{~s}^{2}\left(2 \mathrm{p}_{1 / 2}\right)\left(2 \mathrm{p}_{3 / 2}\right)\left(3 \mathrm{~d}_{5 / 2}\right)$ multiplet. (I've adopted relativistic notation for the sub-shell structure: $n l_{\mathrm{j}}$.) According to the factorization theorem, the cross section for each of these transitions has the form

$$
\sigma\left(2 \mathrm{p}_{1 / 2} \rightarrow 3 \mathrm{~d}_{5 / 2}, \mathrm{E}_{\text {out }}, \Delta \mathrm{E} ; \mathrm{J}=3 / 2-\mathrm{J}_{\mathrm{f}}\right)=\mathrm{A}\left(\mathrm{p}_{1 / 2} \rightarrow \mathrm{d}_{5 / 2} ; \mathrm{J}=3 / 2-\mathrm{J}_{\mathrm{f}}\right) \mathrm{R}\left(2 \mathrm{p}_{1 / 2} \rightarrow 3 \mathrm{~d}_{5 / 2}, \mathrm{E}_{\text {out }}, \Delta \mathrm{E}\right),
$$

where $R$ is common to all eight transitions. If we compute $R$ at $\Delta E=\Delta E_{\min }$ and at $\Delta E_{\max }$, and interpolate for the remaining transitions, the eight original calculations are reduced to two. (The angular coefficients are computationally trivial combinations of $3 j$ and $6 j$ symbols). It takes only a moment's reflection to realize the possible savings when this method is applied to more complicated situations, for example, to transitions between a pair of eight level multiplets. As a rough rule of thumb, the computational effort scales with the square of the number of sub-shells in the model, rather than the square of the number of levels.

$$
\left[\left(2 \mathrm{p}_{1 / 2}\right)^{1}\left(2 \mathrm{p}_{3 / 2}\right)^{3}\left(3 \mathrm{~d}_{5 / 2}\right)^{1}\right]_{\mathrm{J}=9 / 2,7 / 2,5 / 2,3 / 2}
$$

$1 / 2,7 / 2,5 / 2,3 / 2$

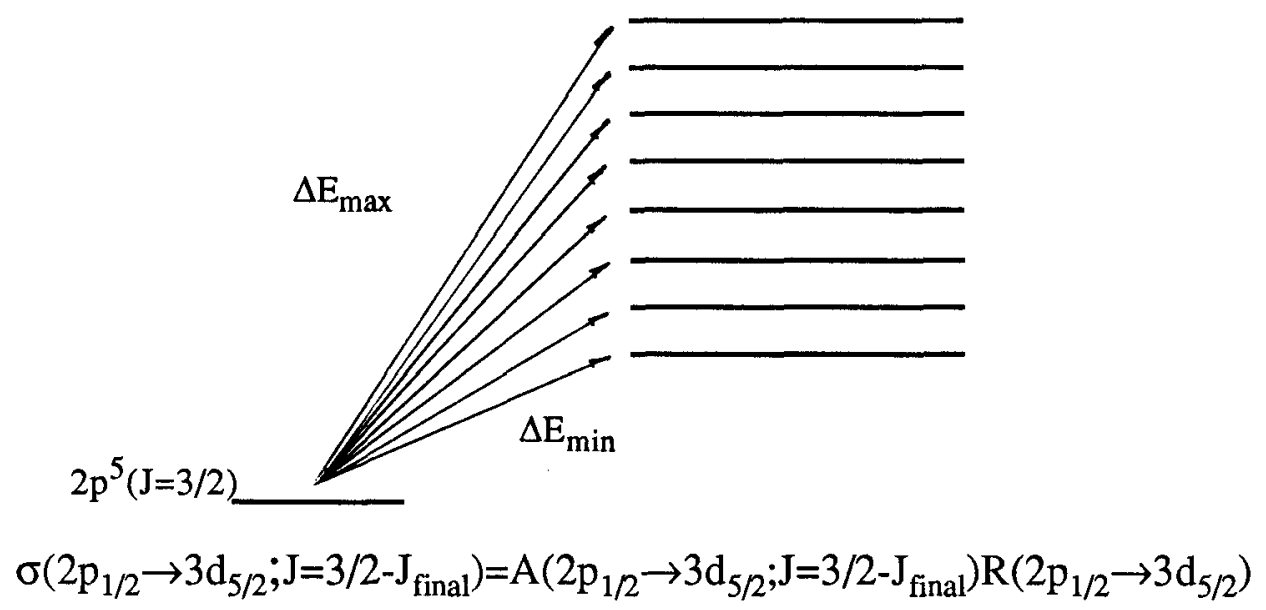

Figure 1 -- Representation of the eight collisional cross sections for exciting the $1 \mathrm{~s}^{2} 2 \mathrm{~s}^{2} 2 \mathrm{p}^{5}(\mathrm{~J}=3 / 2)$ ground level of the fluorine-like ion to levels of the $1 s^{2} 2 s^{2}\left(2 p_{1 / 2}\right)\left(2 p_{3 / 2}\right)\left(3 d_{5 / 2}\right)$ multiplet. Factorization reduces these to an angular coefficient times a radial factor that is common to all eight transitions. By interpolating the value of this factor, all eight cross sections can be obtained from a small subset. 
The fluorine-like example should suggest that the factorization-interpolation method is a boon to low density, coronal modeling as well as to the high density regime. With it, ground state excitation rates for complicated ions -- like fluorine- or oxygen-like -- can be obtained, for a range of temperatures, at minimal cost.

\section{LEVEL-CONFIGURATION SUM RULES FOR COLLISIONAL-RADIATIVE PROCESSES.}

Even the largest models for population kinetics in plasma take into account a very small subset of the accessible atomic levels. Various assumptions and approximations are invoked to justify neglecting high-lying Rydberg levels, autoionizing manifolds and, in fact, entire charge states. But in most cases, the effects of circumscribing the kinetics are not well understood or controlled.

An obvious solution to this problem is to treat a limited set of levels in detail, while adopting a hydrogenic description for a larger set of surrounding configurations. Since it can replace high multiplicity configurations with single effective levels, this approach overcomes the hardship of calculating a host of new rate constants when a manifold is added to the model, and limits the growth of the system of rate equations. Unfortunately, there is not a consistent way to couple hydrogenic "effective" levels to detailed atomic states, and this leads typically to spurious behavior at the boundary between the hydrogenic and detailed descriptions. In particular, not only must one assume that the levels represented by the hydrogenic configuration are statistically populated, but to couple them to detailed levels, statistical assumptions must be made for their population as well, and for the behavior of branching ratios. Furthermore, hydrogenic approximations for rate constants are often very inadequate.

The factorization-interpolation method described in Section 1 underlies an alternative approach to expanding collisional-radiative models that avoids the inconsistencies of the hydrogenic approximation. This model is based on sum rules that express the total rate or cross section for a transition between a detailed level and a configuration. For collisional excitation, the sum rule follows trivially from Eq. (1):

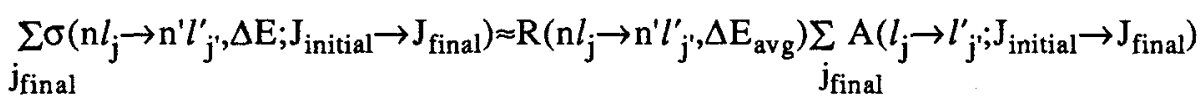

The summation on the right hand side of Eq. (2) can be performed analytically, while the radial factor can be computed in any atomic structure model (hydrogenic, Hartree- or Dirac-Fock, parametric potential, etc.). The only approximation involved in Eq. (2) is the necessity of using an average transition energy, $\Delta \mathrm{E}_{\text {avg }}$, but this is common to all effective level methods. Note that deriving the sum rule depends crucially on being able to factor out of the cross section all dependence on continuum electrons. Analogous factorization theorems have been obtained for collisional ionization, autoionization (Oreg, Goldstein, Bar-Shalom and Klapisch 1988), and radiative transitions (Condon and Shortley 1935, Bauche, Bauche-Arnoult and Klapisch 1988).

The sum rules represent exact, analytic expressions for the rate of a process summed over final states, and, conversely, for the inverse process averaged over initial states. Together with a statistical ansatz for the distribution of population within a summed 
configuration, they provide a consistent and efficient -- not to mention elegant -- way to collapse high multiplicity configurations down to effective levels, and to couple these levels to detailed atomic structure.

Sum rules fit neatly and unobtrusively at any point in a collisional-radiative model. They are as computationally efficient as the hydrogenic approach, but far more accurate and require no assumptions about branching ratios into detailed levels. They have specific applications to treating Rydberg series (note that the analytic factor in (2) is independent of n), and to calculating branching ratios, that, after all, are precisely sums over final states. When applied to autoionizing configurations, we obtain a novel configuration average approach to evaluating multi-step, resonant contributions to recombination, ionization and excitation processes, i.e., dielectronic recombination, excitation or ionization followed by autoionization and resonant excitation (Oreg et al. 1988).

\section{A DETAILED CALCULATION OF RESONANT EXCITATION IN FEXVII}

Access to super-computers and the development of powerful, efficient new computer codes has facilitated a deeper level of detail in the atomic modeling we can apply to the collisional-radiative problem. Single configuration LS coupling atomic structure calculations have given way to multiconfigurational, intermediate coupling. Calculations that involved averaging or lumping together the levels of a configuration can now be done using detailed level accounting. This development is most evident in the modeling of resonant processes involving the branching ratios of autoionizing levels. M. H. Chen's recent calculations of dielectronic recombination rates (Chen 1986) and the work of Reed, Chen and Hazi (1988) on resonant excitation in oxygen-like selenium are examples of this trend. The former work, in particular, replaced earlier, less accurate, configuration average, LS coupling results (LaGattuta and Hahn 1983; Roszman 1979; Griffin, Pindzola and Bottcher 1985).

In photo-ionized astrophysical plasmas, ionization of inner-shell electrons can lead to the emission of one or more additional Auger electrons, and this process is governed, also, by branching ratios for autoionizing levels. Calculations of the rate at which this process populates excited levels of neon- and fluorine-like ions of iron will be necessary to properly analyze high resolution spectra of accretion disk sources.

Even in the case of the simpler solar coronal plasmas, the importance of resonant excitation and dielectronic recombination for understanding FeXVII spectra has been recognized. In a comprehensive analysis of this problem, Smith et al. (1985) calculated resonant excitation rates and the dielectronic satellites of neon-like iron. There is the suggestion as well that dielectronic recombination from FeXVIII is important in the formation of FeXVII lines in the corona (Liedahl et al. 1988).

Resonant excitation of neon-like ions, particularly of the $2 p^{5} 3 \mathrm{~s}$ levels whose direct excitation cross sections are small, is of interest in the laboratory plasma community as well. Determining the population of these levels is a challenge to modelers because under almost any plasma conditions, it is populated both directly and indirectly, by both collisional and cascade processes. And the record in accurately modeling the intensity of

the $2 p^{6}-2 p^{5} 3 s$ lines is spotty at best. Relative intensities in the $2 p-3 s$ lines far exceeding what would be expected based on comparative oscillator strength have been observed in both laser-produced and vacuum spark plasmas (Goldstein et al. 1987; Finkenthal et al. 
1988) as demonstrated in Figure 2. One hypothesis for these anomalies is that they represent contributions from a low temperature phase where recombination and/or resonant excitation is important.

Figure 3 shows a SOLEX spectrum (Rugge and McKenzie 1985). The wavelength region covered is the analog in iron of the titanium spectrum in 2(b). Here the M2 line to the long wavelength side of $G$ appears owing to the much lower electron density. Otherwise, there is much in common between the laboratory and solar spectra, including the signal strength of the $2 \mathrm{p}-3 \mathrm{~s}$ lines. As an example of an application of detailed level accounting, we have performed a new calculation of resonant excitation rates for the $2 \mathrm{p}^{5} 3 \mathrm{~s}(\mathrm{~J}=0,1,1,2)$ levels in FeXVII. Our goal is to improve on the original estimates of Smith et al. for this astrophysically important process, and to help gauge its impact on laboratory plasma analysis. (A recent calculation by Omar and Hahn, 1988, uses a nonrelativistic, LS coupling, term average method that is unlikely to be adequate.)

Figure 4 is a reminder of how resonant excitation works. An electron with the correct energy colliding with a neon-like ion in its ground state can excite a resonance that is essentially a doubly excited sodium-like level. Once a free electron is captured this way, it can de-excite in several ways, depending on the total energy of the doubly excited state. If the energy is below that of the first excited neon-like level (the $2 \mathrm{p}^{5} 3 \mathrm{~s}, \mathrm{~J}=2$ ), as depicted in figure $4(\mathrm{a})$, the state either autoionizes back to $2 \mathrm{p}^{6}$ plus a free electron -- a contribution to elastic scattering, or it radiatively relaxes, forming a stable sodium-like state, a process called dielectronic recombination. At higher incident electron energies, as shown in figure 4(b),resonances are excited that, in addition to the deexcitation pathways enumerated in 4(a), have the option of autoionizing to excited neon-like levels.

(a)

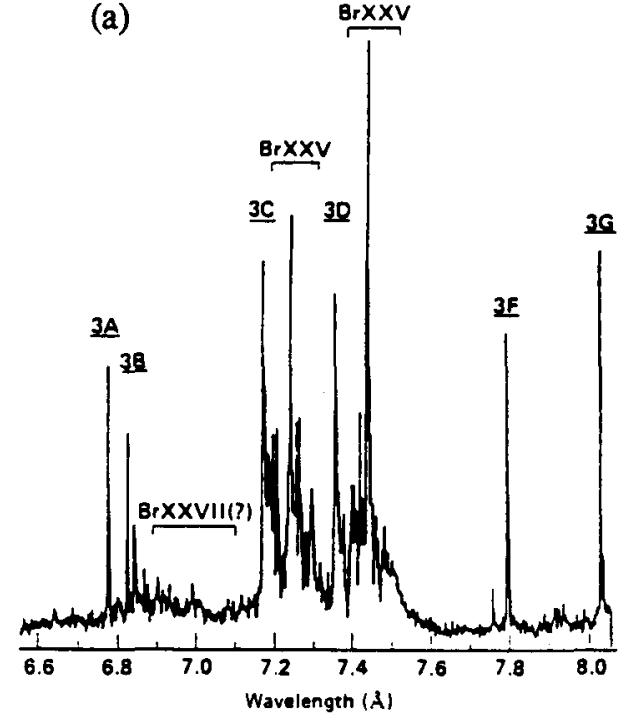

(b)

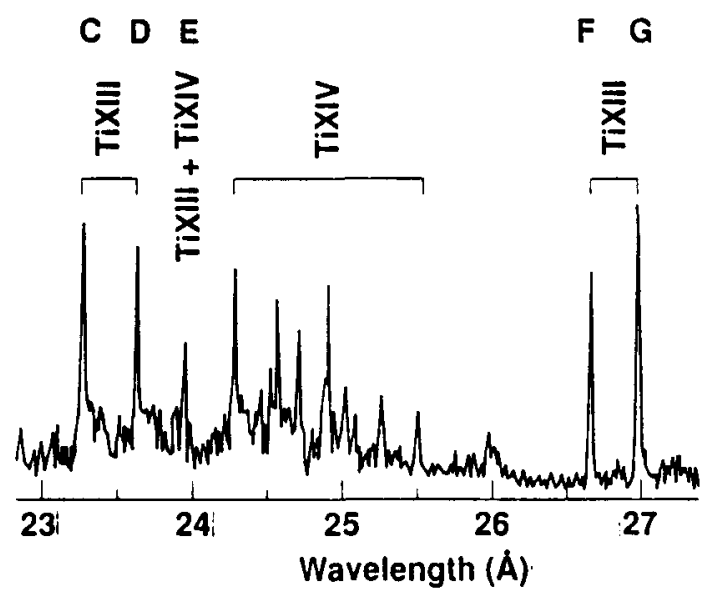

Figure 2 -- (a)Time-integrated spectrum of neon-like bromine produced by $3 \times 10^{13} \mathrm{~W} / \mathrm{cm}^{2}$ from $.53 \mu \mathrm{m}$ laser. $\mathrm{N}=3-\mathrm{n}=2$ resonance lines are labeled $3 \mathrm{~A}, \mathrm{~B}, \mathrm{C}, \mathrm{D}, \mathrm{F}, \mathrm{G}$. The latter two are anomalously strong 2p-3s transitions (Goldstein et al. 1987). (b) Time-integrated snectrum of neon-like titanium produced in a vacuum spark (Finkenthal et al. 1988). 


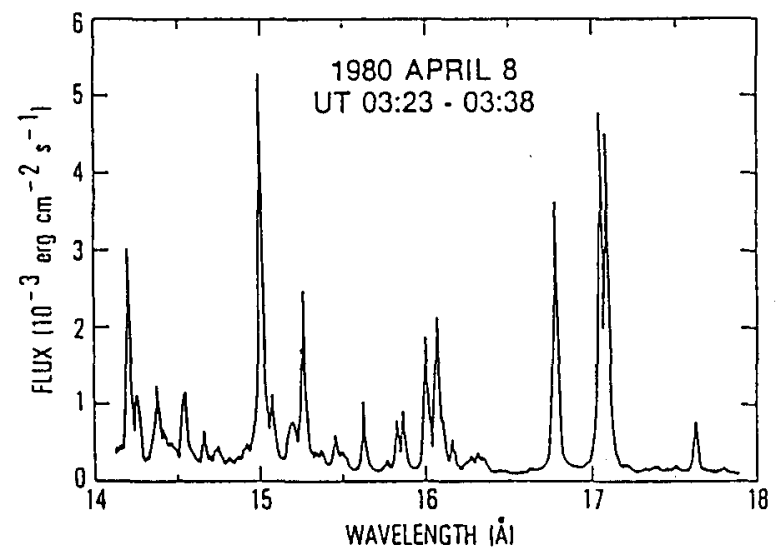

(a)

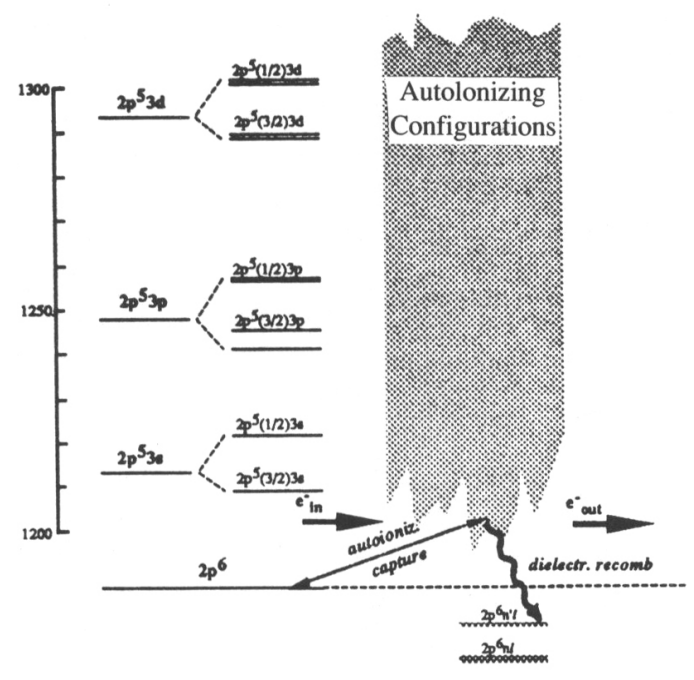

(b)
Figure 3 -- Solar flair spectrum from Rugge and McKenzie (1985) showing the neon-like iron $2 \mathrm{p}-3 \mathrm{~d}$ resonance lines at $15.0,15.3$ and $15.5 \AA$, and the $2 \mathrm{p}-3 \mathrm{~s}$ lines at 16.8 and $17.0 \AA$. The direct excitation rates for the latter are smaller by a factor of 30 than that of the $15 \AA$ line. The extra line at $17.1 \AA$ is the $2 \mathrm{p}^{6}$ $2 \mathrm{p}^{5} 3 \mathrm{~s}(\mathrm{~J}=2)$ E2 transition. This line is absent in the high density laser-produced and vacuum spark plasmas shown in Fig. 2.

$$
\frac{2 p^{6} 3 d}{2 p^{6} 3 p}
$$

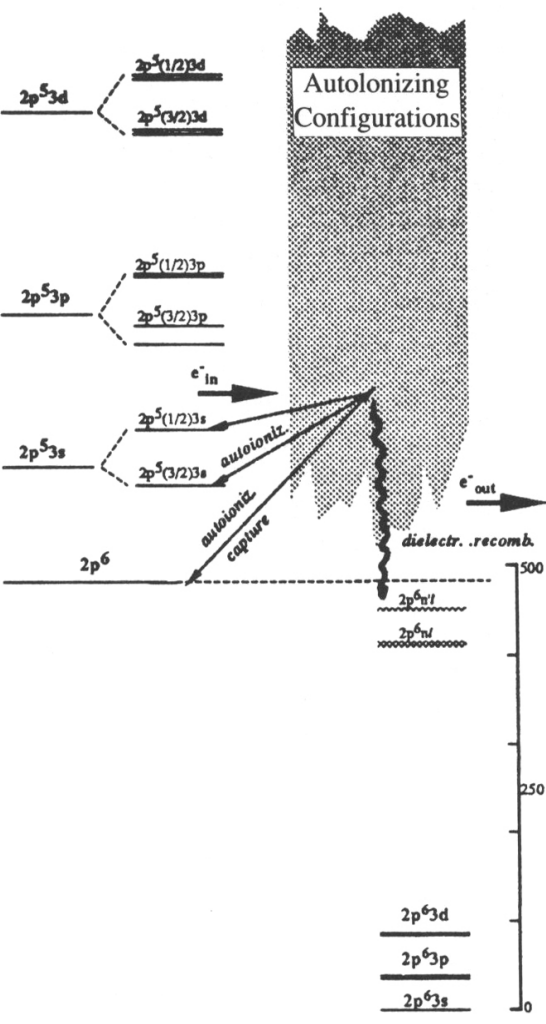

Figure 4 -- (a) Free electrons with energy below threshold for excitation of the neon-like $2 \mathrm{p}^{5} 3 \mathrm{~s}$ level undergo either elastic scattering or dielectronic recombination. (b) Electrons with energy above threshold have additional deexcitation channels available: resonant excitation of neon-like excited states. (Energy scales are in $\mathrm{eV}$.) 
This sequence of events is a "resonant" contribution to collisional excitation since it leaves a free electron and an excited neon-like level in the final state. In the isolated resonance approximation, the rate coefficient for this process is given by

$$
\mathrm{A}^{\text {autoioniz. }} \text { (level } \rightarrow \text { final) }
$$

$$
\begin{aligned}
& \mathrm{R}^{\text {res.exc. }}{ }_{\text {(final) }}=\sum \sum \mathrm{R}_{\text {capt. }}^{\text {cap6 }} \rightarrow \text { level) } \\
& \mathrm{C}_{\text {auto }} \text { levele }_{\text {auto }} \quad \sum_{\mathrm{j}}\left[\mathrm{A}^{\text {autoioniz. }}(\text { level } \rightarrow \mathrm{j})+\mathrm{A}^{\mathrm{rad} .}(\text { level } \rightarrow \mathrm{j})\right]
\end{aligned}
$$

where "final" is a neon-like level and the sodium-like "level" belongs to the autoionizing configuration $\mathrm{C}_{\text {auto }}$. The capture rate is obtained by detailed balancing the autoioization rate, and the last factor is the branching ratio for "level." The sum in the denominator can generally be truncated to include only the dominant radiative decay branch.

The importance of accounting explicitly for each level (both "final" and "level") in Eq. (3) can be seen by noting that the branching ratio is very sensitive to the opening of new autoionization channels with increasing energy. The intricate interleaving of sodium-like doubly-excited and neon-like levels makes it essential to retain the full detail of the level structure to obtain accurate branching ratios. A good example of this effect is found in the

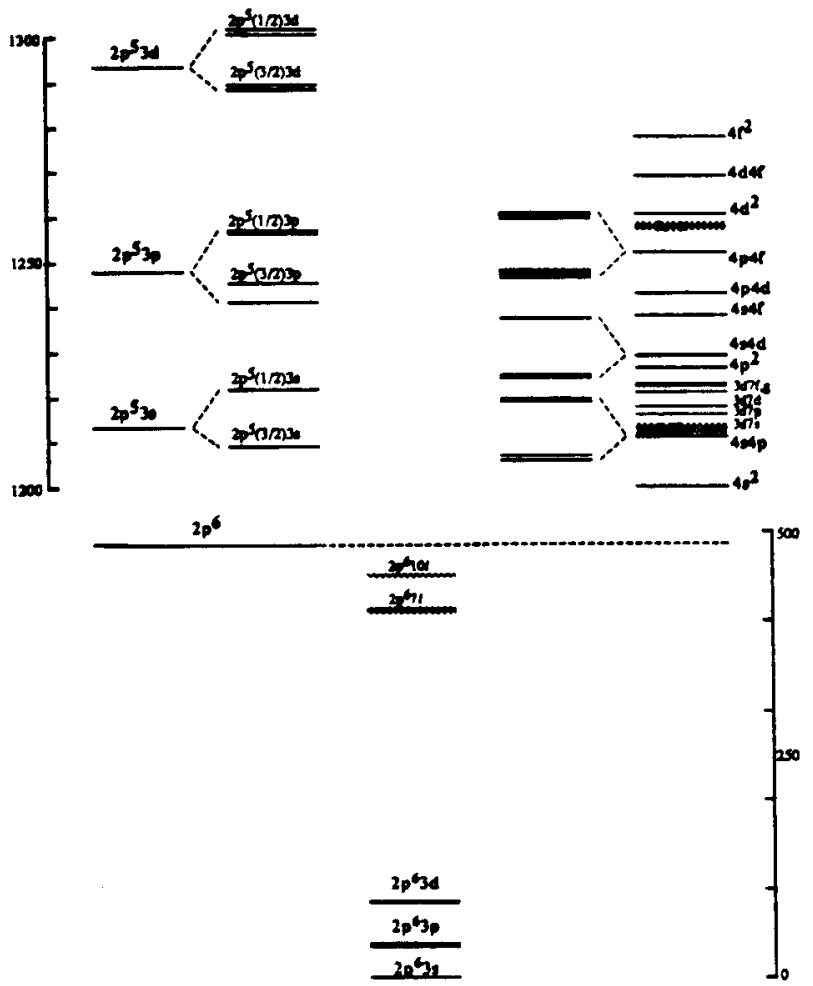

Figure 5 -- Schematic energy level diagram for neon-like and sodium-like iron. The (broken) energy scale is in $\mathrm{eV}$. On the left are the shown the $2 \mathrm{p}^{5} 3 l$ configurations of the neon-like ion, broken down as far as their relativistic sub-shell structure. The branching into individual levels is not shown. On the right are the configurations of the form $2 \mathrm{p}^{5} 4 l 4 l$. To convey an idea of how these manifolds interleave with the neon-like, several have been broken into sub-configurations. Note that the actual detailed level structure used in the calculation is not shown here.

$2 \mathrm{p}^{5} 4 l 4 l^{\prime}$ configurations, which, as shown in Figure 5, are spread over many autoionizing channels. Furthermore, detailed level accounting is necessary to avoid having to make 
statistical assumptions about population distribution at some point in the calculation. Such assumptions are always hazardous in calculations involving fluorescent yields where selection rules play an important role (Chen, Crasseman and Matthews 1975).

The autoionizing configurations included in the outer summation in Eq. (3), and the radiative and autoionizing channels included in the branching ratios for the present calculations are listed in Table 1. Besides applying detailed level accounting for all the configurations listed, this calculation differs from its predecessors in including the $2 p^{5} 3 p$ and $2 \mathrm{p}^{5} 3 \mathrm{~d}$ autoionization channels for $\mathrm{C}_{\mathrm{auto}}=2 \mathrm{p}^{5} 4 l 4 l$. As shown by figure (5), these channels cannot be neglected for these configurations, and they have the effect of reducing the partial contribution to the rate of excitation of the $2 \mathrm{p}^{5} 3 \mathrm{~s}$ by more than a factor of two. Atomic structure, including energy levels, autoionization rates and Einstein coefficients, were obtained in the relativistic parametric potential model (Klapisch et al. 1977).

In light of our significant disagreement with previous results, a comment is in order on consistency checks of this calculation. The same set of data was used to calculate partial dielectronic recombination rates for the $2 \mathrm{p}^{5} 3 l 3 l^{\prime}$ and $2 \mathrm{p}^{5} 4 l 4 l$ configurations. The results fell within $20 \%$ of the calculation of Chen (1986). In addition, an independent calculation was solicited from Chen (1988) of the $2 \mathrm{p}^{5} 4 l 4 l$ contribution to resonant excitation of the $2 \mathrm{p}^{5} 3 \mathrm{~s}$ levels. Using his multiconfigurational Dirac-Fock code (MCDF) and the detailed level accounting scheme, he obtained results that agreed with the present calculation to better than $5 \%$. Finally, the convergence of the series in $n$ and $l$ were verified; no extrapolation beyond $\mathrm{n}=14$ was performed for the $3 \mathrm{dn} l l^{\prime}$ and $3 \mathrm{pn} l$ 'series. The latter series had not quite converged, and we estimate that our results in this case may undershoot the converged value by up to $15 \%$.

The results of this calculation, at an electron temperature of $200 \mathrm{eV}$, are presented in Table 2 , along with the results quoted by Smith et al. (1985), broken down by final state and autoionizing series. The importance of detailed level accounting is evident in the reduction found in rates by factors ranging from 3 to 5 . (The factor of 10 between the $4 l 4 l^{\prime}$ contributions is reduced to 5 when the a correction is made for the $2 p^{5} 3 p$ channel not included in the earlier calculation.)

\begin{tabular}{llc}
\hline \multicolumn{1}{c}{$\mathrm{C}_{\text {auto }}$} & \multicolumn{1}{c}{$\begin{array}{c}\mathrm{C}_{\mathrm{Ne}} \\
\text { (autoion. channels) }\end{array}$} & $\begin{array}{c}\mathrm{C}_{\mathrm{Na}} \\
\text { (rad. channels) }\end{array}$ \\
\hline $2 \mathrm{p}^{5} 3 \mathrm{dn} l, l \leq 4,6 \leq \mathrm{n} \leq 14$ & $2 \mathrm{p}^{6}, 2 \mathrm{p}^{5} 3 \mathrm{~s}, 2 \mathrm{p}^{5} 3 \mathrm{p}$ & $2 \mathrm{p}^{6} \mathrm{n} l(\mathrm{E} 1), 2 \mathrm{p}^{6} 3 \mathrm{~d}(\mathrm{E} 1)$ \\
$2 \mathrm{p}^{5} 3 \mathrm{pn} l, l \leq 4,8 \leq \mathrm{n} \leq 14$ & $2 \mathrm{p}^{6}, 2 \mathrm{p}^{5} 3 \mathrm{~s}$ & $2 \mathrm{p}^{6} 3 \mathrm{p}(\mathrm{E} 1), 2 \mathrm{p}^{6} \mathrm{n} l(\mathrm{E} 1, \mathrm{E} 2)$, \\
& & $2 \mathrm{p}^{5} 3 \mathrm{sn} l(\mathrm{E} 1)$ \\
$2 \mathrm{p}^{5} 4 l 4 l^{\prime}, l \leq 3$ & $2 \mathrm{p}^{6}, 2 \mathrm{p}^{5} 3 \mathrm{~s}$, & $2 \mathrm{p}^{6} 4 l(\mathrm{E} 1), 2 \mathrm{p}^{5} 3 l 4 l^{\prime}(\mathrm{E} 1)$ \\
& $2 \mathrm{p}^{5} 3 \mathrm{p}, 2 \mathrm{p}^{5} 3 \mathrm{~d}$ & \\
\hline
\end{tabular}

Table 1 -- The autoionizing configurations included in the present calculation, with their Auger and radiative decay branches. Configuration interaction $(\mathrm{CI})$ among autoionizing levels was included to the extent that for each $n$, the energy was diagonalized on the space of all $1(\leq 4)$. CI between the $2 \mathrm{p}^{5} 3 \mathrm{pn} l$ and $2 \mathrm{p}^{5} 3 \mathrm{sn} l$ was included to allow for E1 decays to the $2 p^{5} n l$. All levels of the $2 p^{5} 4 l 4 l^{\prime}$ were included in a single structure calculation. 


\begin{tabular}{|c|c|c|c|c|c|c|c|c|}
\hline \multirow{3}{*}{$\begin{array}{l}2 \mathrm{p}^{5} 3 \mathrm{~s} \\
\text { state }\end{array}$} & \multicolumn{6}{|c|}{ AUTOIONIZING SERIES } & \multirow{2}{*}{\multicolumn{2}{|c|}{$\begin{array}{l}\text { TOTAL RATE } \\
\times 10^{-13} \mathrm{~cm}^{3} \mathrm{sec}^{-1}\end{array}$}} \\
\hline & \multicolumn{2}{|c|}{$2 p^{5} 3 p n l$} & \multicolumn{2}{|c|}{$2 \mathrm{p}^{5} 3 \mathrm{dn} l$} & \multicolumn{2}{|c|}{$2 p^{5} 4 l 4 l^{\prime}$} & & \\
\hline & $\mathrm{A}$ & $\mathrm{B}$ & A & $\mathrm{B}$ & $\bar{A}$ & $\mathrm{~B}$ & $\mathrm{~A}$ & $\mathrm{~B}$ \\
\hline $\mathrm{J}=2$ & 5.72 & 1.51 & 12.2 & 7.14 & 31.0 & 2.94 & 49.0 & 11.6 \\
\hline $\mathrm{J}=1$ & 13.4 & 4.40 & 10.7 & 4.61 & 20.7 & 2.06 & 44.8 & 11.1 \\
\hline $\mathrm{J}=0$ & 0.86 & 0.18 & 3.12 & 1.11 & 6.70 & 1.08 & 10.7 & 2.37 \\
\hline $\mathrm{J}=1$ & $\underline{8.65}$ & $\underline{4.27}$ & $\underline{8.17}$ & $\underline{4.43}$ & $\underline{18.3}$ & $\underline{2.25}$ & 35.1 & 11.0 \\
\hline All J & 28.6 & 10.4 & 34.2 & 17.3 & 76.7 & 8.33 & 140. & 36.1 \\
\hline
\end{tabular}

Table 2 -- Partial and total resonant excitation rate coefficients $\left(10^{-13} \mathrm{~cm}^{3} \mathrm{sec}^{-1}\right)$ for the $2 p^{5} 3 \mathrm{~s}$ levels of FeXVII. Values for this calculation (B) are at an electron temperature of $200 \mathrm{eV}$ and for $\mathrm{n} \leq 14$, while those of Smith et al. (1985) (A) are at $217 \mathrm{eV}$ and include an extrapolation of $\mathbf{n}$ to 100 .

\section{ACKNOWLEDGEMENT}

I'd like to acknowledge my co-workers, J. Oreg, A. Bar-Shalom, M. Klapisch and A. Osterheld, and many very helpful consultations with M. H. Chen. Work performed under the auspices of the U.S. Department of Energy by Lawrence Livermore National Laboratory under contract \#W-7405-Eng-48.

\section{REFERENCES}

Bar-Shalom, A., Klapisch, M. and Oreg, J. 1988, Phys. Rev. A, 38, 1773.

Bauche, J., Bauche-Amoult, C. and Klapisch, M. 1988, Transition Arrays in the Spectra of Ionized Atoms, to appear in Advances in Atomic and Molecular Physics.

Chen, M. H., Crasseman, B., Matthews, D. L. 1975, Phys. Rev. Lett., 34, 1309.

Chen, M. H. 1986, Phys. Rev. A, 34, 1073.

Chen, M. H. 1988, private communication.

Condon, E. U. and Shortley, G. H. 1935, Theory of Atomic Spectra (London : Cambridge University Press)

Finkenthal, M., Stutman, D., Mandelbaum, P., Osterheld, A. L., Goldstein, W. H. and Chen, M. H. 1988, "Electron Density Measurement of a Pre-pinched Vacuum Spark Plasma Using Soft X-ray Titanium and Vanadium Emission," UCRL-99351, to appear in J. Phys. B: At. Mol. Phys.

Goldstein, W. H., Walling, Bailey, J., Chen, M. H., Fortner, R., Klapisch, M., Phillips, T. and Stewart, R. E. 1987, Phys. Rev. Lett., 58, 2300.

Goldstein , W. H. and Reed, K. J. 1986, in Conference on Atomic Processes in Hot Dense Plasma, Jerusalem (unpublished).

Griffin, D. C., Pindzola, M. S. and Bottcher, C. 1985, Phys. Rev. A, 31, 568.

Klapisch, M., Schwob, J. L., Fraenkel, B. S. and Oreg, J. 1977, J. Opt. Soc. Am., 61, 148.

LaGattuta, K. and Hahn, Y. 1983, Phys. Rev. A, 27, 1675.

Liedahl, D., Kahn, S., Osterheld, A. L. and Goldstein, W. H., in preparation.

Omar, G. and Hahn, Y. 1988, Phys. Rev. A, 37, 1983.

Oreg, J. 1971, J. Math. Phys., 12, 1018.

Oreg, J. 1975, Ph.D. Thesis (Hebrew University).

Oreg, J., Goldstein, W.H., Bar-Shalom, A. and Klapisch, M. 1988, UCRL-98456, "SumRules for the Collisional Radiative Model," submitted. to Phys. Rev. A.

Reed, K. J., Chen, M. H. and Hazi, A. U. 1988, "Autoionizing Resonances in Electron Impact Excitation of Oxygen-like Selenium," UCRL-98374, submitted to Phys. Rev. A.

Roszman, L. J. 1979, Phys. Rev. A, 20, 673.

Rugge, H. R. and McKenzie, D. L. 1985, Ap. J., 297, 338.

Smith, B. W., Raymond, J. C., Mann, J. B. and Cowan, R. D. 1985, Ap. J., 298, 898. 


\section{DISCUSSION-W. Goldstein}

B. Smith: You began your talk by describing some shortcuts you took in computing atomic rates. I am not sure these represent an advance. It is certainly not safe to compute populations of levels within configurations according to statistical weights.

I would also like to correct one or two errors you made in describing our work. We did include configuration interaction in all detailed calculations. Quantum defect methods were used only to obtain the n-dependence of autoionization rates.

W. Goldstein: In the case of excitation cross-sections, the factorization is exact, whereas the interpolation in threshold energy that this factorization allows, can be handled with any degree of precision required. Since this technique opens the door to modeling systems that were not that were not tractable previously - those in which, say hundreds, or more excitation rates are relevant - it certainly represents an advance.

The sum rules are also exact, given the necessity of using configuration average transition energies. This would be the only approximation involved, then, in using the sum rules in the calculation of branching ratios (sums over final state). I certainly agree that it is dangerous to use them, if the intention is to distribute this rate statistically amongst individual levels. The accuracy of such a procedure must be considered on a case-by-case basis. (The accuracy will depend on electron density, ionic charge, principal quantum number, etc).

With regard to your calculation of resonant excitation, the absence of any reference to configuration interaction led me to the assumption that it was not included. Also, the paper seemed to suggest that, for all but a handful of doubly-excited configurations, a quantum-defect estimate was adopted for the total autionization rate from the group of levels with an " $n$ " electron above a neon-like core, to each neon-like level. This rate, I assumed, was distributed statistically amongst the "l" values of the Rydberg configuration. 